\title{
Successful Percutaneous Intervention to Acute Myocardial Infarction Presenting With Typical Chest Pain in Transplanted Heart
}

\author{
Ulas Bildirici, MD; Umut Celikyurt, MD; Ertan Ural, MD; Tayfun Sahin, MD; \\ Ahmet Vural, MD; Cem Koz, MD*; Dilek Ural, MD
}

\begin{abstract}
Allograft coronary artery disease (CAD) is the main limiting factor of the long-term survival in cardiac transplant recipients. It is present in approximately $50 \%$ of patients at 5 years post-transplant. Allograft CAD might result in congestive heart failure, ventricular arrhythmias and sudden cardiac death. Acute coronary syndrome is a rare presentation of allograft CAD, because symptoms are usually atypical or absent as a result of cardiac denervation. However, it should be reminded that reinnervation of the transplanted heart might occur, and angina pectoris could be felt as a sign of an acute coronary syndrome. We present a 27 -year-old female cardiac transplant patient with an acute myocardial infarction, who was successfully treated with percutaneous transluminal coronary angioplasty. (Circ J 2009; 73: 2166-2168)
\end{abstract}

Key Words: Acute myocardial infarction; Angioplasty; Transplantation

$A$

llograft coronary artery disease $(\mathrm{CAD})$ is the main limiting factor of the long-term survival in heart transplanted patients. It develops in approximately $50 \%$ of patients at 5 years after transplantation. Allograft CAD might result in congestive heart failure, ventricular arrhythmias and sudden cardiac death. Acute coronary syndrome is a rare presentation of allograft CAD. ${ }^{1}$ Symptoms are usually atypical or absent as a result of cardiac denervation. We present a 27 -year-old female patient with an acute myocardial infarction (AMI) who presented with typical chest pain and was successfully treated with percutaneous transluminal coronary angioplasty (PTCA).

\section{Case Report}

A 27-year-old female patient had a history of restrictive cardiomyopathy. She had undergone heart transplantation 5 years ago at another institution. The donor was a 43-yearold male patient without any previous $\mathrm{CAD}$ and known risk factors. Echocardiography revealed normal left ventricular wall motion and an ejection fraction of $60 \%$ after the transplantation. Four years after transplantation, clinical ventricular tachycardia episodes and severe bradycardia were detected. Echocardiographic examination showed severe hypokinesis in apical segments, hypokinesis in mid and apical segments of anterior and lateral walls, decreased left ventricular ejection fraction (42\%) and significant inter- and intraventricular conduction delay without any akinetic area. Biventricular pacemaker-implantable cardioverter defibrillator implantation was done.

Coronary angiography, which was done 1 year before index presentation revealed diffuse distal narrowing in multiple coronary arteries and allograft vasculopathy was considered. Intravenous bolus nitrate was given during coronary angiography, and the diameters of the coronary arteries were dilated without any residual critical stenosis.

She presented to our hospital with severe retrosternal chest pain of $2 \mathrm{~h}$ duration. Physical examination revealed a blood pressure of $80 / 60 \mathrm{mmHg}$, heart rate was 80 beats/min and respiratory rate was 22 breaths per min. Other physical examination findings were normal. Electrocardiography revealed pacemaker rhythm, which was non-diagnostic for AMI (Figure 1). The first troponin level that was measured at the time of admission was negative. An echocardiography revealed apical akinesia. Aspirin, clopidogrel, unfractionated heparin and statin treatment was initiated. The emergent coronary angiography revealed total occlusion of the proximal segment of left anterior descending (LAD) artery with an ectatic structure (Figure 2A). There was a 70\% stenosis at the circumflex artery. The right coronary artery was rudimentary. An angioplasty with a size of $1.5 \mathrm{~mm} / 20 \mathrm{~mm}$ balloon was performed and antegrade flow was obtained. A diagonal branch also appeared at the level of occlusion after the first balloon inflation. A second wire was inserted to this diagonal branch, and the size of a $4.0 \mathrm{~mm} / 18 \mathrm{~mm}$ balloon was inflated up to 18 atmosphere in LAD. Thrombolysis in myocardial infarction III flow was obtained, and stent implantation was not done because of the ectasia in this vessel segment (Figure 2B). Maximum CKMB and troponin I values in hospital follow-up were $437 \mathrm{mg} / \mathrm{dl}$ and $23.9 \mathrm{ng} / \mathrm{ml}$, respectively.

During follow up, the patient was asymptomatic for 5

Received March 6, 2008; revised manuscript received December 11, 2008; accepted December 17, 2008; released online February 13, 2009

Kocaeli University Medical Faculty, Department of Cardiology, Interventional Cardiology Research and Application Unit, Kocaeli, *Gulhane Military Medical Academy, Faculty of Medicine, Department of Cardiology, Ankara, Turkey

Mailing address: Umut Celikyurt, MD, Kocaeli University Medical Faculty, Department of Cardiology, Interventional Cardiology Research and Application Unit, 41380, Kocaeli, Turkey. E-mail: ycelikyurt@gmail.com

All rights are reserved to the Japanese Circulation Society. For permissions, please e-mail: cj@j-circ.or.jp 

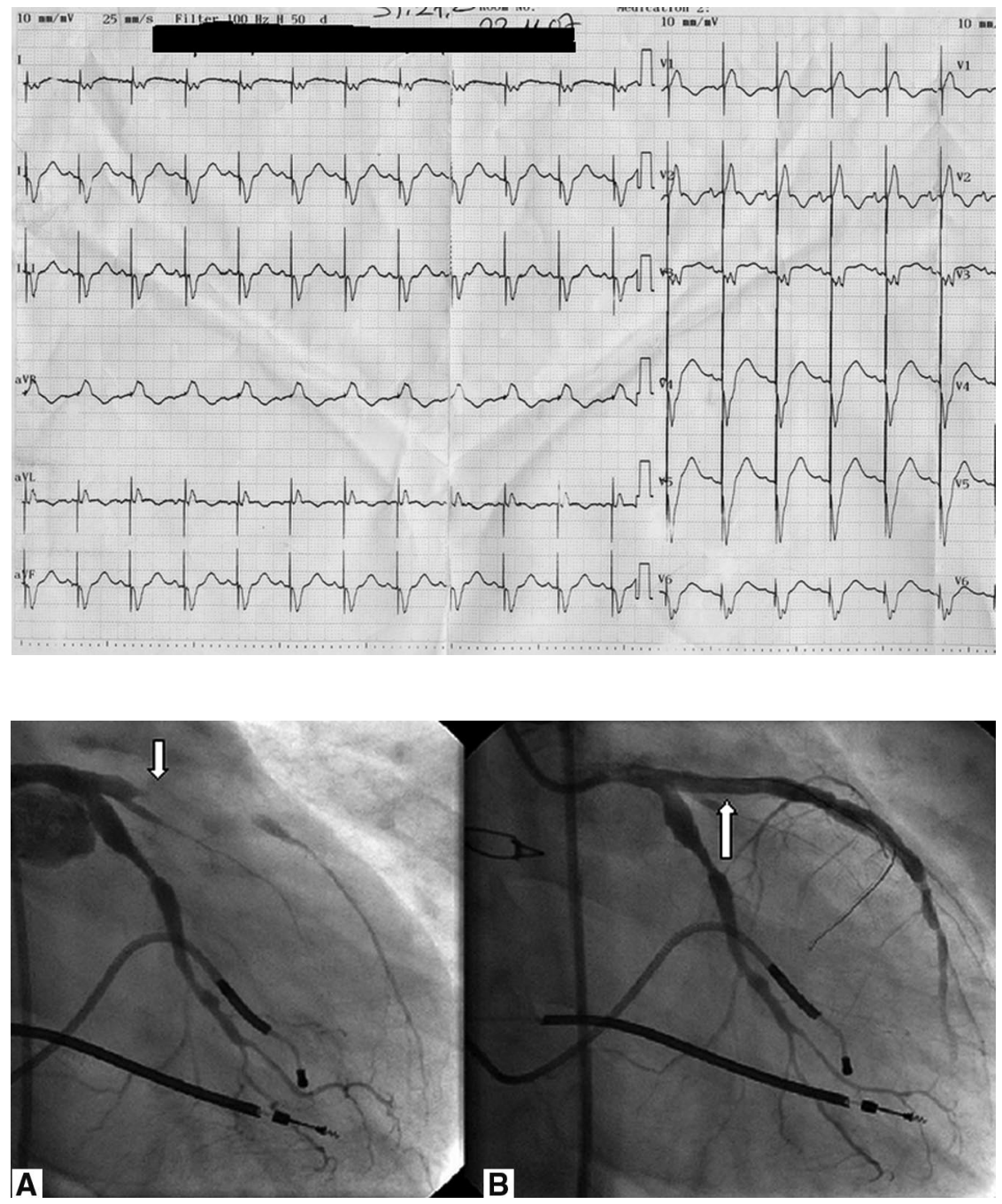

Figure 1. Electrocardiography during myocardial infarction.

Table. The Characteristics of the Patients

\begin{tabular}{|c|c|c|c|}
\hline & Fazio et $\mathrm{al}^{2}$ & Di Cori et al ${ }^{4}$ & Yamada et al $^{5}$ \\
\hline Age of patient (years) & 42 & 67 & 56 \\
\hline Gender & M & M & M \\
\hline Underlying disease (reason for transplantation) & Ischemic cardiomyopathy & Ischemic cardiomyopathy & Dilated cardiomyopathy \\
\hline Time interval between diagnosis and myocardial infaction & 10 years & 7 years & 16 years \\
\hline Culprit coronary artery lesion & LAD & LAD & LAD \\
\hline Treatment & Only PTCA & $3.5 \times 18 \mathrm{~mm}$ stent implantation & $\begin{array}{l}\text { Cypher } 3.5 \times 23 \mathrm{~mm} \text { followed by } \\
\text { balloon dilatation }(4 \times 8 \mathrm{~mm})\end{array}$ \\
\hline
\end{tabular}

LAD, left anterior descending; PTCA, percutaneous transluminal coronary angioplasty.

months and echocardiography revealed an apical akinesis in a small segment.

\section{Discussion}

Allograft vasculopathy is an accelerated form of CAD. It develops in approximately $50 \%$ of patients at 5 years after transplantation. Multiple factors such as immunosupressive drugs, viral infections, donor age and presence of an acute rejection are believed to be involved in the pathophysiology of allograft vasculopathy. ${ }^{1-3}$

The diagnosis of an acute coronary syndrome in cardiac transplant recipients is difficult as a consequence of auto- nomic denervation of the transplanted heart. ${ }^{4}$ For this reason, acute coronary syndrome rarely presents as myocardial infarction in these patients. Myocardial infarction is usually diagnosed in the chronic or subacute phase and most common presents as heart failure symptoms or ventricular arrhythmias. Therefore primary angioplasty is performed rarely in cardiac transplant recipients.

We could find 3 case reports in the literature, which were presented with typical clinical picture of an acute coronary syndrome in heart transplant recipients (Table).2,4,5 Our patient also presented with typical angina pectoris. This condition can be explained with reinnervation of the transplanted heart, which occurs in approximately $10-30 \%$ of 
patients. ${ }^{6}$ The exact mechanism of the reinnervation process is not known.

The other difficulty in the diagnosis of AMI in our patient was the presence of a pacemaker rhythm on the electrocardiography. The close echocardiographic follow-up and the knowledge of her previous myocardial functions were the critical points of the diagnosis of AMI in this particular case, which allowed us to compare the myocardial functions between the examinations.

Optimal treatment modalities of acute coronary syndrome in cardiac transplant recipients are not clear. Although in a study comparing long-term results of stenting and angioplasty in allograft vasculopathy stenting has a lower restenosis rate at 6 and 12 months follow-up, 5 year restenosis rates were similar. ${ }^{7}$ We performed PTCA without stenting. Stent implantation was not done because of the ectasia in this vessel segment, the diameter of the vessel was above $4.0 \mathrm{~mm}$. In addition, drug eluting or bare metal stent implantation in the ectatic vessel might result in malposition, if the dilatation of the vessel continues.

\section{Conclusion}

Acute coronary syndrome rarely presents with typical angina pectoris in cardiac transplant recipients. However, it should be reminded that reinnervation of the transplanted heart might occur and angina pectoris could be felt as a sign of an acute coronary syndrome. Echocardiography is very helpful, especially in the presence of undiagnostic electrocardiographic findings such as pacemaker rhythm.

\section{Disclosure}

There is no actual or potential conflict of interest of the authors.

\section{References}

1. Syeda B, Roedler S, Schukro C, Yahya N, Zuckermann A, Glogar D. Transplant coronary artery disease: Incidence, progression and interventional revascularization. Int J Cardiol 2005; 104: 269-274.

2. Fazio G, Sutera L, Vernuccio D, Fazio M, Novo S. Symptomatic acute myocardial infarction in a patient bearer of heart transplantation following ischemic heart disease. Intern J Cardiol 2008; 124: 233-236.

3. Vigano M, Tuzcu M, Bena R, Boissonnat P, Haverich A, Hill J, et al. Prevention of acute rejection and allograft vasculopathy by everolimus in cardiac transplants recipients: A 24-month analysis. J Heart Lung Transplant 2007; 26: 584-592.

4. Di Cori A, Petronio AS, Gemignani C, Zucchelli G, Di Bello V, Mariani M. Symptomatic acute myocardial infarction in a cardiac transplant recipient successfully treated with primary coronary angioplasty: Evidence of prognostic importance of chest pain after cardiac transplantation. J Heart Lung Transplant 2005; 24: 1146-1149.

5. Yamada A, Ishii Y, Kakizawa Y, Ishige A, Ueno H, Omichi C, et al. Angina pectoris in patients who underwent stent implantations for severe atherosclerotic coronary lesions 16 years after heart transplantation. Kyobu Geka 2007; 11: 969-973.

6. Aranda JM Jr, Hill J. Cardiac transplant vasculopathy. Chest 2000; 118: $1792-1800$

7. Simpson L, Lee EK, Hott BJ, Vega DJ, Book WM. Long-term results of anjioplasty vs stenting in cardiac transplant recipients with allogaft vasculopathy. J Heart Lung Transplant 2005; 24: 1211 - 1217. 